\title{
Glycemic Control in Hospitalized Patients with Diabetes Receiving Corticosteroids Using a Neutral Protamine Hagedorn Insulin Protocol: A Randomized Clinical Trial
}

Ameer Khowaja · Jamil B. Alkhaddo - Zaighum Rana • Lisa Fish

Received: May 11, 2018 / Published online: June 30, 2018 (C) The Author(s) 2018

\section{ABSTRACT}

Introduction: Hospitalized patients with diabetes receiving corticosteroids are at risk of developing hyperglycemia and related complications. This study evaluated a neutral protamine Hagedorn (NPH) insulin-based protocol in improving glycemic control in hospitalized patients receiving corticosteroids.

Methods: This was a randomized, prospective, non-blinded study in an inpatient setting involving patients with diabetes who were hospitalized and receiving prednisone $\geq 10 \mathrm{mg}$ per day or equivalent. High dose corticosteroids

Enhanced digital features To view enhanced digital features for this article go to https://doi.org/10.6084/ m9.figshare.6633050.

Electronic supplementary material The online version of this article (https://doi.org/10.1007/s13300018-0468-3) contains supplementary material, which is available to authorized users.

A. Khowaja · J. B. Alkhaddo $\cdot$ Z. Rana $\cdot$ L. Fish Division of Diabetes, Endocrinology and

Metabolism, University of Minnesota, Minneapolis, MN, USA

J. B. Alkhaddo

The Center for Diabetes and Endocrine Health, Allegheny Health Network, Pittsburgh, PA, USA

\section{A. Khowaja $(\bowtie) \cdot$ L. Fish}

Center for Diabetes and Endocrinology, Hennepin

Healthcare System, Minneapolis, MN, USA

e-mail: ameer.khowaja@hcmed.org group (prednisone $>40 \mathrm{mg} /$ day or equivalent) received NPH insulin $0.3 \mathrm{U} / \mathrm{kg}$ between 0600 and 2000 hours if eating or $0.2 \mathrm{U} / \mathrm{kg}$ between 2000 and 0600 hours if not eating. Low dose corticosteroids group (prednisone 10-$40 \mathrm{mg} /$ day or equivalent) received $0.15 \mathrm{U} / \mathrm{kg}$ between 0600 and 2000 hours if eating or $0.1 \mathrm{U} /$ $\mathrm{kg}$ between 2000 and 0600 hours if not eating. Primary outcome measure was mean blood glucose level measured pre-meal and at bedtime for days $1-5$.

Results: Mean blood glucose level was lower in the intervention $(n=29)$ than in the usual care $(n=31)$ group $[226.12$ vs. $268.57 \mathrm{mg} / \mathrm{dL}$, respectively, (95\% CI for difference -63.195 to $-21.695), p<0.0001]$. Significant differences in mean glucose level were noted at fasting [170.96 vs. $221.13 \mathrm{mg} / \mathrm{dL}$, respectively, (95\% CI for difference -72.70 to -27.63$), p<0.0001]$ and pre-lunch [208 vs. $266.48 \mathrm{mg} / \mathrm{dL}$, respectively, $(95 \%$ CI for difference -86.61 to $-30.36), p<0.0001]$.

Conclusion: In hospitalized patients with diabetes receiving corticosteroids, an NPH insulinbased protocol improves glycemic control.

Trial Registration: ClinicalTrials.gov Identifier: NCT01970241.

Funding: Eli Lilly and Company.

Keywords: Diabetes mellitus; DM; Neutral protamine Hagedorn insulin; NPH; Steroids 


\section{INTRODUCTION}

Corticosteroids cause hyperglycemia by increasing insulin resistance; and when they are used to treat illnesses in persons with diabetes, significant hyperglycemia results. Possible mechanisms of corticosteroid-induced hyperglycemia include increased availability of cortisol [1], decreased postprandial glucose uptake, glycogen synthesis by skeletal muscles [2], and increase hepatic gluconeogenesis [3]. In a study of healthy volunteers given corticosteroids, a doubling of plasma insulin concentration and a $50 \%$ reduction in insulin sensitivity were found [4]. In hospitalized patients, $50-60 \%$ of patients without diabetes have at least one glucose level over $200 \mathrm{mg} / \mathrm{dL}$ while on corticosteroids and essentially all patients with a diagnosis of diabetes have significant hyperglycemia $[5,6]$.

Corticosteroids used to treat hospitalized patients may result in acute hyperglycemia, suboptimal glycemic control, longer hospital stays, and increased morbidity and mortality $[6,7]$. Use of corticosteroids in the hospital is predominantly for chronic obstructive pulmonary disease (COPD), as a component of chemotherapy, and after transplantation [6]. When corticosteroids are used to treat COPD there is an increased risk of death which rises by $10 \%$ for each $18 \mathrm{mg} / \mathrm{dL}$ increase in blood glucose [8]. For patients with acute lymphoblastic leukemia, hyperglycemia occurred in $37 \%$ and those patients had shorter survival (29 vs. 88 months) [9]. In the setting of solid organ transplantation, hyperglycemia with glucose over $200 \mathrm{mg} / \mathrm{dL}$ is associated with an increased risk of rejection and infection [10-12].

Prevalence of diabetes among hospitalized patients is estimated to range from $12.4 \%$ to $26 \%$ [13]. Efforts to adjust inpatient diabetes regimens to mitigate the rise in plasma glucose related to corticosteroids have been challenging. Patients receiving corticosteroids are being treated for variety of illnesses with different corticosteroid preparations in terms of dose, frequency, duration of action, and time of administration. These issues have made corticosteroid-induced hyperglycemia a difficult problem to study, and the current practice of adjusting usual insulin regimens is often ineffective.

Several studies have suggested that neutral protamine Hagedorn (NPH) insulin may be useful in treating corticosteroid-induced hyperglycemia in the setting of diabetes $[14,15]$. Continuous glucose monitoring in patients with COPD treated with daily prednisolone showed an increase in glucose concentration throughout the day with a return to baseline glucose levels between 2000 and 0800 hours [16]. This is a time course suited for $\mathrm{NPH}$ insulin which has an approximately 4-h peak and 18-h duration of action [17].

We conducted a prospective, randomized, non-blinded study in hospitalized diabetes patients comparing an NPH insulin-based protocol utilizing type and dose of corticosteroid, patient's oral intake status, and time of the day added to the patient's pretreatment basal-bolus insulin regimen for the management of corticosteroid-induced hyperglycemia.

\section{METHODS}

\section{Study Design and Patient Population}

This was a prospective, randomized, non-blinded, parallel arm study conducted at Hennepin County Medical Center, Minneapolis, MN. Study protocol was approved by the institutional review board, Minneapolis Medical Research Foundation. All procedures performed in studies involving human participants were in accordance with the ethical standards of the institutional and/or national research committee and with the 1964 Helsinki declaration and its later amendments or comparable ethical standards. Informed consent was obtained from all individual participants included in the study.

Patients were recruited from August 2014 until April 2016. The study population included patients between 18 and 79 years of age with diabetes, hospitalized for at least $48 \mathrm{~h}$, and receiving supraphysiologic doses of corticosteroids (prednisone $\geq 10 \mathrm{mg} /$ day or equivalent) orally or intravenously. Data was collected for up to 5 days of hospitalization. Patients with 
critical illness, eGFR $<30 \mathrm{~mL} / \mathrm{min} / 1.73 \mathrm{~m}^{2}$, hepatic dysfunction (ALT $>2$ times the upper limit of normal reference range), corticosteroid doses less than prednisone $10 \mathrm{mg} /$ day or equivalent, and/or duration of corticosteroid therapy for less than $48 \mathrm{~h}$ were excluded (Supplemental Fig. 1).

Prospective patients were identified by tracking inpatient corticosteroid dispensation by the hospital pharmacy. Electronic health records of those patients were reviewed for the diagnosis of diabetes and screened for eligibility criteria. Patients were then randomized to either intervention (NPH insulin-based protocol) group or usual care group using a standard randomization table.

\section{Insulin Protocol}

All patients in both groups received their outpatient insulin regimen of basal-bolus, premixed or basal only. Bolus insulin was held if patients were not eating and premixed insulin was switched to basal only proportionately to the basal part of the premixed insulin. Non-insulin diabetes drugs in both groups were held. Patients in both groups with hemoglobin a1c $>9.0 \%$ at the time of enrollment received $0.3 \mathrm{U} / \mathrm{kg} /$ day of insulin glargine. Moreover, patients in both arms received a correction dose of insulin aspart using a factor of $2 \mathrm{U}$ for every $50 \mathrm{mg} / \mathrm{dL}$ over $200 \mathrm{mg} / \mathrm{dL}$ and was increased up to $3 \mathrm{U}$ for every 50 over $200 \mathrm{mg} / \mathrm{dL}$ after the first $24 \mathrm{~h}$ of the study period if blood glucose continued to remain above $200 \mathrm{mg} / \mathrm{dL}$ (Supplemental Fig. 1).

In the intervention group, patients were categorized as receiving either high or low dose corticosteroids. NPH insulin dose was calculated accordingly (Supplemental Fig. 2). High dose corticosteroid was defined as prednisone $\geq 40 \mathrm{mg} /$ day, hydrocortisone $\geq 160 \mathrm{mg} /$ day, methylprednisolone $\geq 32 \mathrm{mg} / \mathrm{day}$, and dexamethasone $\geq 6 \mathrm{mg} /$ day. Low dose corticosteroid was defined as prednisone between 10 and $40 \mathrm{mg}$ or equivalent dose of hydrocortisone, methylprednisolone, and dexamethasone. NPH insulin was given at the same time as the corticosteroid dose administration when dosing was QD, BID, and TID. If dosing was more frequent than TID, then NPH dose was administered at 0800, 1600, and 2200 hours.

Patients in the control arm were continued on basal insulin once a day with or without bolus insulin with meals along with the correction scale as described above. Dose titration for corticosteroid was done per the discretion of the primary inpatient healthcare providers.

\section{Sample Size Calculation}

We determined that to detect a $20 \%$ difference in pre-meal mean blood glucose between the two groups with power of $80 \%$ and confidence interval (CI) of $95 \%$, we would need a sample size of 60 patients.

\section{Statistical Analysis}

Analysis was performed using Statistical Package for Social Sciences (IBM SPSS 12 v24). To determine the distribution of baseline and clinical variables, the Shapiro-Wilk test was used. An independent two-sample $t$ test was used for normally distributed outcomes whereas the Mann-Whitney $U$ test was used for blood glucose values which were not normally distributed. Two-sided 95\% CI was used to assess the statistical significance between the intervention and usual care group. Pearson correlation and chi-square tests were used to test the linear relationship between C-peptide and other baseline variables.

\section{Outcome Measures}

The primary outcome measure was the mean point of care (POC) blood glucose as measured four times daily (pre-meals and bedtime) for days $1-5$ in the intervention and usual care group. Secondary outcome measures included incidence of hypoglycemia (POC glucose $<70 \mathrm{mg} / \mathrm{dL}$ ), percentage of POC glucose measurements between 70 and $180 \mathrm{mg} / \mathrm{dL}$, incidence of hyperglycemia defined as POC glucose $180-300 \mathrm{mg} / \mathrm{dL}, 300-400 \mathrm{mg} / \mathrm{dL}$, and $>400 \mathrm{mg} / \mathrm{dL}$, difference in mean glucose on the first and last day of the study between the 
intervention and control groups, difference in mean insulin dose, and length of stay.

\section{RESULTS}

Sixty patients completed the study (29 in intervention and 31 in usual care group) (Fig. 1). Indications for corticosteroids were cancer-related treatment or autoimmune, musculoskeletal, and pulmonary conditions. Both groups were comparable in terms of baseline characteristics (Table 1).
Mean POC glucose was lower in the intervention group than the usual care group [226.12 vs. $268.57 \mathrm{mg} / \mathrm{dL}$, respectively, (95\% CI for difference -63.195 to -21.695$)$, $p<0.0001]$. Significant differences in mean glucose level were noted at fasting [170.96 vs. $221.13 \mathrm{mg} / \mathrm{dL}$, respectively, (95\% CI for difference -72.70 to -27.63$)$, $p<0.0001]$ and prelunch [208 vs. $266.48 \mathrm{mg} / \mathrm{dL}$, respectively, (95\% CI for difference -86.61 to -30.36$)$, $p<0.0001]$ but not pre-dinner $[283.17$ vs. $307.77 \mathrm{mg} / \mathrm{dL}$, respectively, (95\% CI for difference -55.52 to 6.33), $p=0.118$ ] and at bedtime

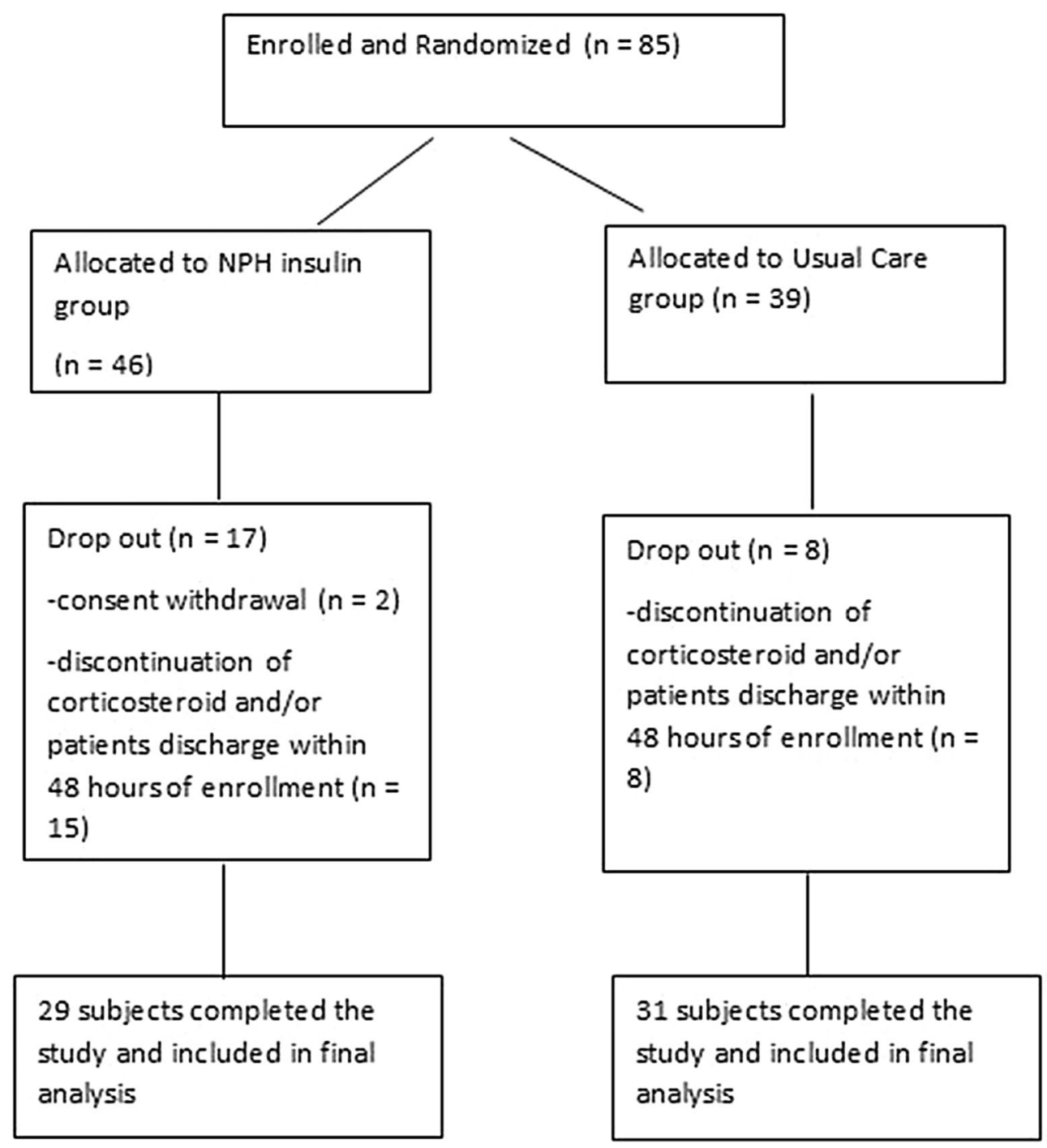

Fig. 1 CONSORT diagram showing the flow of participants 
Table 1 Baseline characteristics of patients in intervention and usual care group

\begin{tabular}{lllll}
\hline Variables & Usual care $(\boldsymbol{n}=\mathbf{3 1})$ & Intervention $(\boldsymbol{n}=\mathbf{2 9})$ & $\mathbf{9 5 \%}$ CI for difference of mean & $\boldsymbol{p}$ values \\
\hline Age $($ years $)$ & 53.94 & 57.83 & -2.68 to 10.47 & 0.241 \\
$\mathrm{BMI}\left(\mathrm{kg} / \mathrm{m}^{2}\right)$ & 35.08 & 37.26 & -2.88 to 7.24 & 0.392 \\
GFR $\left(30 \mathrm{~mL} / \mathrm{min} / 1.73 \mathrm{~m}^{2}\right)$ & 105.10 & 88.10 & -39.43 to 5.44 & 0.135 \\
Creatinine $(\mathrm{mg} / \mathrm{dL})$ & 0.92 & 1.03 & -0.08 to 0.305 & 0.251 \\
ALT $(\mathrm{U} / \mathrm{L})$ & 26.97 & 28.66 & -7.63 to 11.01 & 0.718 \\
Hemoglobin alc $(\%)$ & 8.85 & 8.11 & -1.63 to 0.162 & 0.106 \\
C-peptide $(\mathrm{ng} / \mathrm{mL})$ & 6.10 & 5.70 & -2.38 to 1.58 & 0.688 \\
Diabetes duration (years) & 8.33 & 11.21 & -1.84 to 7.60 & 0.227 \\
Admission glucose (mg/dL) & 240.10 & 253.72 & -31.97 to 59.2 & 0.552 \\
Indications for corticosteroid & $n(\%)$ & & & 0.313 \\
Pulmonary & $22(71)$ & $19(65.5)$ & N/A & \\
Cancer & $1(3.2)$ & $5(17.2)$ & & \\
Autoimmune & $6(19.3)$ & $4(13.7)$ & & \\
Musculoskeletal & $2(6.4)$ & $1(3.4)$ & N/A & \\
Type of corticosteroid $n(\%)$ & & & \\
Prednisone & $30(96.7)$ & $25(86.2)$ & $4(13)$ & \\
Dexamethasone & $1(3.2)$ & & \\
\hline
\end{tabular}

[273 vs. $294.07 \mathrm{mg} / \mathrm{dL}$, respectively, (95\% CI for difference -53.63 to 11.49), $p=0.203$ ] (Fig. 2).

Patients in the intervention group had higher proportions of measurements of POC glucose between 70 and $180 \mathrm{mg} / \mathrm{dL}$ than control [33.1\% vs. $19.2 \%$, respectively, (95\% CI for proportion -0.21 to -0.07$), p<0.0001]$ and lower proportions of measurements between 300 and $400 \mathrm{mg} / \mathrm{dL}$ [16.9\% and $27 \%$, respectively, (95\% CI for proportion 0.04 to 0.17 ), $p<0.01]$ whereas there was no difference in glucose level between 180 and $300 \mathrm{mg} / \mathrm{dL}$ $[42.1 \%$ vs. $45.3 \%$, respectively (95\% CI for proportion -0.05 to 0.11 ), $p=0.423$ ] and measurements $>400 \mathrm{mg} / \mathrm{dL} \quad[5.9 \%$ and $8.5 \%$, respectively, (95\% CI for proportion -0.01 to 0.07), $\quad p=0.211]$. Hypoglycemia (glucose $<70 \mathrm{mg} / \mathrm{dL}$ ) was more frequent in the $\mathrm{NPH}$ insulin arm than in the control arm (six episodes in NPH arm vs. zero in control arm); however, three of the episodes occurred prior to study NPH being given and were felt to be related to the home insulin regimen.

Mean insulin added to usual dose per day in the intervention group was significantly higher than in the control group (46.6 vs. $17.4 \mathrm{U} /$ day, $p<0.0001$ ).

There was no difference in POC glucose between the groups on the first day of the study, but glucose level was significantly lower in the intervention group on the last day of the study (Table 2).

There was no difference in mean length of stay between the two groups ( 5.21 vs. 5.23 days, respectively, $p=0.989$ ).

There was a significant correlation between mean glucose and C-peptide $(p=0.009)$ but not between glucose and BMI ( $p=0.372)$ or glucose and hemoglobin a1c $(p=0.648)$. There was no 


\section{Mean POC glucose}



50

\begin{tabular}{|c|c|c|c|c|}
\hline 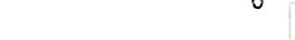 & Fasting & Pre lunch & Pre dinner & Bed time \\
\hline - Usual cares (mg/dl) & 221.13 & 266.48 & 307.77 & 294.07 \\
\hline$\Longrightarrow$ intervention $(\mathrm{m} / \mathrm{dL})$ & 170.96 & 208 & 283.17 & 273 \\
\hline
\end{tabular}

Fig. 2 Mean POC glucose at fasting, pre-meals, and bedtime

Table 2 Difference in mean POC glucose between the groups on first and last day of the study period

\begin{tabular}{llllr}
\hline Mean POC glucose $(\mathbf{m g} / \mathbf{d L})$ & Control $(\boldsymbol{n}=\mathbf{3 1})$ & Intervention $(\boldsymbol{n}=\mathbf{2 9 )}$ & $\mathbf{9 5 \%}$ CI for difference of mean & $\boldsymbol{p}$ value \\
\hline First day of study & 280.85 & 246.91 & -67.98 to 0.11 & 0.051 \\
Last day of study & 253.47 & 197.27 & -92.25 to -20.17 & $<0.01$ \\
\hline
\end{tabular}

correlation between C-peptide and other baseline characteristics including length of stay.

\section{DISCUSSION}

We demonstrated improvement in glycemic control in hospitalized patients with diabetes receiving corticosteroids using an NPH insulinbased protocol. Patients who received NPH insulin got significantly more added insulin per day (47 vs 17 units) to counteract the glucose effect of corticosteroids and the dosing was appropriate for their weight, oral intake status, and dose of corticosteroids.

Other studies which have evaluated NPH insulin for corticosteroid-induced exacerbation of diabetes have shown positive results. A pilot study in patients receiving methylprednisolone daily for cystic fibrosis with NPH dosing based on corticosteroid dose alone showed improvement in glucose levels [18]. Grommesh et al. using a similar protocol that was not weightadjusted achieved significance in a subset of patients, as a result of most patients enrolled not having preexisting diabetes [15]. A report from the Netherlands of add-on NPH insulin compared to sliding scale in 26 diabetes patients receiving cyclical chemotherapy showed an increase in time-in-target for patients receiving the NPH insulin without symptomatic hypoglycemia [19]. Two studies evaluated using basal-bolus regimens for corticosteroid-treated patients. Ruiz de Adana et al. studied 53 type 2 patients in a pulmonary ward who were receiving multiple doses of intermediate-acting steroids daily and compared NPH given three times a day to once a day glargine in patients 
who were hospitalized for 8-10 days. Ruiz de Adana et al. did not find a difference in glucose control [20]. Burt et al. studied general medicine patients taking prednisolone and found that despite aggressive adjustment of basal-bolus insulin they were not able to adequately control the glucose levels in the afternoon and evening [21]. More recently Lakhani et al. demonstrated that a predetermined protocol-driven insulin dosing regimen leads to significant improvement in corticosteroid-induced hyperglycemia at fasting, pre-meals, and bedtime [22]. Their study protocol used three different insulins (regular human insulin, NPH, and glargine) in the intervention group depending upon the type of corticosteroid used whereas our protocol utilized only NPH insulin administered at the time of corticosteroid dosing (daily, BID, or TID). Our purpose in using NPH insulin was to determine if a protocol utilizing a single insulin type improves glycemic control equivalently and hence can be integrated conveniently into clinical practice. Moreover, in case of abrupt discontinuation of corticosteroids, duration of $\mathrm{NPH}$ insulin compared to glargine minimizes the risk of hypoglycemia.

The NPH insulin protocol used in this study considers key variables affecting patient's glucose level including dose and frequency of corticosteroids, patient's caloric intake, and patient's weight, which were not utilized in previous studies [15]. Moreover, we did not restrict our study population to patients receiving corticosteroids for specific etiologies, which makes it more generalizable. Two-thirds of study participants received corticosteroids for pulmonary disease while the remainder were treated for inflammatory disorders or cancer. In our cohort, patients were not noted to be on medications other than glucocorticoid with significant influence on glucose metabolism. Categorization of corticosteroids into high and low dose offers a degree of individualization as well as a way to reduce the insulin dosing readily as the corticosteroid dose is tapered. Most patients in this study had type 2 diabetes mellitus and one-third were not on insulin as outpatients. They were a diverse group with preadmission insulin dosing ranging from 0 to over 300 units/day, and were suboptimally controlled as indicated by hemoglobin a1c of about $8 \%$. While hypoglycemic episodes were more frequent in the intervention group, they were mild, and occurred mainly in patients who were having hypoglycemia on their home regimens. The NPH insulin was administered with the corticosteroid dose, improving safety when corticosteroids are discontinued unexpectedly. The protocol used in this study can also readily be transitioned to the outpatient setting with the patient using an NPH insulin pen and adjusting the dose downward as corticosteroids transition from high to low dose. The patients were continued on their prior insulin regimen in this study, which reduces dosing confusion after the completion of corticosteroid treatment.

Our sample size is relatively small owing to the nature of the study and difficulty in patient recruitment, yet it was adequately powered. A considerable number of patients were excluded from the analysis because of either early discharge from the hospital and/or discontinuation of corticosteroids within $48 \mathrm{~h}$. Our study was open label as blinding of patients and investigators was difficult in an inpatient setting. We used patients' preexisting insulin regimen as is the standard of care at our institution, except for the addition of glargine 0.3 units/kg for patients with admission hemoglobin a1c levels over 9\%. Adjusting the outpatient regimen to balance background insulin to $50 \%$ of the total would have likely reduced hypoglycemia. Moreover, considering recent advancements in continuous glucose monitoring (CGM) technologies, it would have been beneficial especially in preventing hypoglycemia and severe hyperglycemia. Lastly, increasing the NPH dosing daily for patients with elevated pre-supper glucose levels would probably have improved glucose results, and should be considered in future protocol modifications.

\section{CONCLUSION}

In hospitalized patients with diabetes receiving corticosteroids, an NPH insulin-based protocol adjusted to account for corticosteroid dose, 
frequency, patient's weight, and oral intake can improve glycemic control and prevent potential hyperglycemia-related complications. Further studies are needed to evaluate adjustment in $\mathrm{NPH}$ insulin dosing for corticosteroid-induced hyperglycemia in inpatient as well as outpatient settings.

\section{ACKNOWLEDGEMENTS}

We thank the study personnel and patients for their contribution and participation in the study. Authors would like to acknowledge Bilquis Khan for providing statistical support.

Funding. Sponsorship for this study and article processing charges were funded by Eli Lilly and Company. All authors had full access to all of the data in this study and take complete responsibility for the integrity of the data and accuracy of the data analysis.

Authorship. All named authors meet the International Committee of Medical Journal Editors (ICMJE) criteria for authorship for this article, take responsibility for the integrity of the work as a whole, and have given their approval for this version to be published.

Disclosures. Ameer Khowaja, Jamil B Alkhaddo, Zaighum Rana and Lisa Fish have nothing to disclose.

Compliance with Ethics Guidelines. All procedures performed in studies involving human participants were in accordance with the ethical standards of the institutional and/or national research committee and with the 1964 Helsinki declaration and its later amendments or comparable ethical standards. Informed consent was obtained from all individual participants included in the study.

Data Availability. The datasets generated and/or analyzed during the current study are available from the corresponding author on reasonable request.
Open Access. This article is distributed under the terms of the Creative Commons AttributionNonCommercial 4.0 International License (http:// creativecommons.org/licenses/by-nc/4.0/), which permits any noncommercial use, distribution, and reproduction in any medium, provided you give appropriate credit to the original author(s) and the source, provide a link to the Creative Commons license, and indicate if changes were made.

\section{REFERENCES}

1. Van Raalte DH, Ouwens DM, Diamant M. Novel insights into glucocorticoid-mediated diabetogenic effects: towards expansion of therapeutic options? Eur J Clin Invest. 2009;39(2):81-93.

2. Ruzzin J, Wagman AS, Jensen J. Glucocorticoid-induced insulin resistance in skeletal muscles: defects in insulin signalling and the effects of a selective glycogen synthase kinase-3 inhibitor. Diabetologia. 2005;48(10):2119-30.

3. Perez A, Jansen-Chaparro S, Saigi I, Bernal-Lopez MR, Miñambres I, Gomez-Huelgas R. Glucocorticoid-induced hyperglycemia. J Diabetes. 2014;6(1):9-20.

4. Rizza RA, Cryer PE, Haymond MW, Gerich JE. Adrenergic mechanisms for the effects of epinephrine on glucose production and clearance in man. J Clin Invest. 1980;65(3):682-9.

5. Clore JN, Thurby-Hay L. Glucocorticoid-induced hyperglycemia. Endocr Pract. 2009;15(5):469-74.

6. Donihi AC, Raval D, Saul M, Korytkowski MT, DeVita MA. Prevalence and predictors of corticosteroid-related hyperglycemia in hospitalized patients. Endocr Pract. 2006;12(4):358-62.

7. Umpierrez GE, Isaacs SD, Bazargan N, You X, Thaler LM, Kitabchi AE. Hyperglycemia: an independent marker of in-hospital mortality in patients with undiagnosed diabetes. J Clin Endocrinol Metab. 2002;87(3):978-82.

8. Baker EH, Janaway CH, Philips B, et al. Hyperglycaemia is associated with poor outcomes in patients admitted to hospital with acute exacerbations of chronic obstructive pulmonary disease. Thorax. 2006;61(4):284-9.

9. Weiser MA, Cabanillas ME, Konopleva M, et al. Relation between the duration of remission and hyperglycemia during induction chemotherapy for 
acute lymphocytic leukemia with a hyperfractionated cyclophosphamide, vincristine, doxorubicin, and dexamethasone/methotrexate-cytarabine regimen. Cancer. 2004;100(6):1179-85.

10. Ganji MR, Charkhchian M, Hakemi M, et al. Association of hyperglycemia on allograft function in the early period after renal transplantation. Transpl Proc. 2007;39(4):852-4.

11. Wallia A, Parikh ND, Molitch ME, et al. Posttransplant hyperglycemia is associated with increased risk of liver allograft rejection. Transplantation. 2010;89(2):222-6.

12. Wallia A, Gupta S, Garcia C, et al. Examination of implementation of intravenous and subcutaneous insulin protocols and glycemic control in heart transplant patients. Endocr Pract. 2014;20(6):527-35.

13. Clement S, Braithwaite SS, Magee MF, et al. Management of diabetes and hyperglycemia in hospitals. Diabetes Care. 2004;27(2):553-91.

14. Seggelke SA, Gibbs J, Draznin B. Pilot study of using neutral protamine hagedorn insulin to counteract the effect of methylprednisolone in hospitalized patients with diabetes. J Hosp Med. 2011;6(3):175-6.

15. Grommesh B, Lausch MJ, Vannelli AJ, et al. Hospital insulin protocol aims for glucose control in glucocorticoid insulin hyperglycemia. Endocr Pract. 2016;22(2):180-9.

16. Burt MG, Roberts GW, Aguilar-Loza NR, Frith P, Stranks SN. Continuous monitoring of circadian glycemic patterns in patients receiving prednisolone for COPD. J Clin Endocrinol Metab. 2011;96(6):1789-96.
17. Heinemann L, Linkeschova R, Rave K, Hompesch B, Sedlak M, Heise T. Time-action profile of the longacting insulin analog insulin glargine (HOE901) in comparison with those of NPH insulin and placebo. Diabetes Care. 2000;23(5):644-9.

18. Seggelke SA, Gibbs J, Draznin B. Pilot study of using neutral protamine Hagedorn insulin to counteract the effect of methylprednisolone in hospitalized patients with diabetes. J Hosp Med. 2011;6(3):175-6.

19. Gerards MC, de Maar JS, Steenbruggen TG, Hoekstra JB, Vriesendorp TM, Gerdes VE. Add-on treatment with intermediate-acting insulin versus slidingscale insulin for patients with type 2 diabetes or insulin resistance during cyclic glucocorticoidcontaining antineoplastic chemotherapy: a randomized crossover study. Diabetes Obes Metab. 2016;18(10):1041-4.

20. Ruiz de Adana MS, Colomo N, Maldonado-Araque $\mathrm{C}$, et al. Randomized clinical trial of the efficacy and safety of insulin glargine vs. NPH insulin as basal insulin for the treatment of glucocorticoid induced hyperglycemia using continuous glucose monitoring in hospitalized patients with type 2 diabetes and respiratory disease. Diabetes Res Clin Pract. 2015;110(2):158-65.

21. Burt MG, Drake SM, Aguilar-Loza NR, Esterman A, Stranks SN, Roberts GW. Efficacy of a basal bolus insulin protocol to treat prednisolone-induced hyperglycaemia in hospitalised patients. Intern Med J. 2015;45(3):261-6.

22. Lakhani OJ, Kumar S, Tripathi S, Desai M, Seth C. Comparison of two protocols in the management of glucocorticoid induced hyperglycemia among hospitalized patients. Indian J Endocr Metab. 2017;21:836-44. 